\title{
ENTOMOFAUNA ASSOCIADA AO CULTIVO DE SHIITAKE [LENTINULA EDODES (BERK.) PLEGER] NO MUNICÍPIO DE ARROIO DO PADRE, RS, BRASIL
}

\section{E.L.G. da Costa ${ }^{1}$, E. Bernardi2 ${ }^{*}$, J.S. do Nascimento ${ }^{1}$}

${ }^{1}$ Universidade Federal de Pelotas, Instituto de Biologia, Departamento de Microbiologia e Parasitologia, Laboratório de Micologia, CP 354, CEP 96010-900, Pelotas, RS, Brasil. E-mail: elg_dacosta@yahoo.com.br

\author{
RESUMO
}

\begin{abstract}
Durante o cultivo de shiitake em toros de madeira estabelece-se uma diversidade de insetos, em decorrência das condições locais, da fase de desenvolvimento da cultura e/ou do sistema de cultivo. Objetivando-se avaliar a diversidade de insetos associada ao cultivo deste cogumelo cultivado em toros de Eucalyptus spp. foram realizadas avaliações durante a fase de colonização, situado na Cidade de Arroio do Padre, RS. Para isto utilizou-se armadilha luminosa modelo INTRAL AL 012 (12 volts) na captura dos espécimes. Neste período foram detectadas 9 ordens, sobressaindo-se a ordem diptera com maior predominância, apresentando freqüência relativa de $80,57 \%$ e absoluta de 1.393 . Nesta mesma ordem, a família Sciaridae foi representada com maior freqüência absoluta, com 1.085 espécimes.
\end{abstract}

PALAVRAS-CHAVE: Cultivo de shiitake, armadilha luminosa, Diptera, Sciaridae.

\section{ABSTRACT}

INSECTS ASSOCIATED WITH THE SHIITAKE [LENTINULA EDODES (BERK.) PEGLER] CROP IN THE CITY OF ARROIO DO PADRE, RS, BRAZIL. The growing of shiitake on wooden logs attracts a variety of insects depending on the local conditions, the phase of development and the growing system. This study was designed to evaluate the diversity of insects associated to the growing of this mushroom on Eucalyptus spp. logs in the county of Arroio do Padre, RS, by way of evaluations during the colonization phase. A model INTRAL AL 012 (12 volts) luminous trap was used to capture specimens. In this period 9 orders of insects were detected, the order Diptera predominating, presenting a relative frequency of $80.57 \%$ and absolute frequency of 1,393 . In this order, the family Sciaridae was found in the greatest absolute frequently, there being 1,085 specimens.

KEY WORDS: Growing shiitake, luminous trap, Diptera, Sciaridae.

\section{INTRODUÇÃO}

Um dos cogumelos comestíveis mais consumidos no mundoé o shiitake [Lentinulaedodes (Berk.) Pleger], pertencente à classe Basideomycete (CHANG; MILES, 1989). O cultivo deste teve inicio na China, sendo introduzido no Japão por intermédio de cultivadores chineses. Posteriormente, a cultura expandiu-se para outros países (RoYSE et al., 1985; SАВOTA, 1996).

O shiitake tem sido cultivado em toros de madeira ou em serragem suplementada com nutrientes (cultivo axênico), principalmente nos países orientais como o Japão, Taiwan, China, Tailândia, Coréia e Malásia, como também em países do ocidente como EUA, Canadá, México e Brasil (LeAtham, 1985; PrZYBYLOWICZ;
Donoghue, 1990; Oie, 1991; Mata et al., 1998; Chang, 1999, CHANG, 2002; BuENo et al., 2004).

No Brasil, a cultura ainda é realizada em pequena escala de produção e de maneira rudimentar, muitas vezes adaptando instalações já existentes, usando a técnica de inoculação em toros de Eucalyptus spp. (Quimio et al., 1995). O Estado de São Paulo destaca-se como o maior produtor nacional, onde muitos descendentes de imigrantes orientais produzem o cogumelo em sistemafamiliar de pequenas propriedades (BONONI et al., 1995). Neste sistema de cultivo, um dos principais problemas sanitários verificados tem sido a incidência de contaminações, tanto no cogumelo como nos toros incubados, geralmente fungos competidores e causadores de doenças, seguidos por bactérias,

${ }^{2}$ Universidade Federal de Pelotas, Faculdade de Agronomia, Pelotas, RS, Brasil.

"Bolsista CNPq - Programa de Pós-Graduação em Agronomia. 
vírus, nematóides e insetos. Entretanto, as maiores perdas na produção de cogumelos, principalmente em regiões tropicais, são atribuídas ao ataque de insetos especialmente em ambientes não controlados, podendo ultrapassar 20\% (BONONI, 1995; EIRA et al., 1997; BuENo et al., 2004).

Figueiredo; Mucci (1985) relataram pragas em Agaricus campestris com destaque para ordem Diptera, famílias Sciaridae, Phoridae, Cecidomyiidae e também, mas com menor expressão, a ordem Lepidoptera e a subclasse Collembola. JáAL-AMIDI (1995)em trabalho desenvolvido na República da Irlanda, destacou como principais famílias, Sciaridae e Phoridae da ordem Diptera. Assim, o conhecimento sobre os espécimes de insetos que ocorrem na cultura de cogumelos permite a identificação prévia de insetos-pragas para posterior controle (BONHAM et al., 2002; GASTON et al., 2002; JACTEL et al., 2002).

Este trabalho teve como objetivo conhecer a diversidade de insetos associada ao cultivo de shiitake inoculadoem toros de eucalipto, noSul do RioGrandedoSul.

\section{MATERIAIS E MÉTODOS}

O presente artigo constou de coletas de insetos, capturados em armadilha luminosa, na Cidade de Arroio do Padre, RS. Para isto, foi utilizada uma área de produção de shiitake, com 3 meses de existência, localizada na referida cidade. Os toros, para fins de produção de shiitake, foram previamente inoculados conforme metodologia descrita porEIRA;MINHONI (1997) e incubados em galpão de madeira (6 m x $12 \mathrm{~m})$, com irrigação diária. Após o aparecimento de grandequantidade delarvas nos toros foi realizada uma pulveriza- ção com extrato de cinamomo (Melia azedarach L.), com a seguinte formulação: $1 \mathrm{~kg}$ de folhas trituradas e diluídas em 10 L de água, na proporção de 1:10.

A captura dos espécimes foi realizada no período de junho a outubro de 2005, durante a fase de incubação da cultura. A técnica de captura utilizada foi através de armadilha luminosa modelo INTRAL AL 012 (12 volts), provida de lâmpada fluorescente(PINTO et al., 2000; LARANJEIRO, 2003). Foram realizadas 10 coletas, sendo em média, uma a cada 15 dias. A armadilha foi instalada sempre no final da tarde, aleatoriamente sobre os toros na fase de incubação e recolhida após $12 \mathrm{~h}$.

Os espécimes, depois de capturados, foram transferidos para recipientes contendo álcool $70 \%$ até o período de quantificação e identificação. Estas foram realizadas noLaboratório Experimental deMicologia/ Departamento de Microbiologia e Parasitologia/Instituto deBiologia/UniversidadeFederal dePelotas, onde foram triados e quantificados. Alguns foram montados e catalogados para acondicionamento no Laboratório de Insetos localizado no referido Departamento.

\section{RESULTADOS E DISCUSSÃO}

Durante o período de exposição das armadilhas foram capturados 1.729 espécimes, todos pertencentes à classe Insecta. Foram identificadas 10 ordens: Diptera, Hymenoptera, Lepidoptera, Coleoptera, Homoptera, Thysanoptera, Trichoptera, Hemiptera, Dermaptera e Orthoptera. Destas, apenas Diptera, Hymenoptera e Lepidoptera ocorreram em todas as coletas. A ordem Diptera foi a que representou maior quantidade de espécimes capturados (Tabela 1).

Tabela 1 - Total de espécimes capturados no período de incubação de Lentinula edodes (shiitake) cultivado em toros de eucalipto, no Município de Arroio do Padre, RS, 2005.

\begin{tabular}{|c|c|c|c|c|c|c|c|c|c|c|c|}
\hline \multirow[t]{2}{*}{ Ordens } & \multicolumn{10}{|c|}{ Coletas } & \multirow[t]{2}{*}{ Total } \\
\hline & 1 & 2 & 3 & 4 & 5 & 6 & 7 & 8 & 9 & 10 & \\
\hline Diptera & 211 & 221 & 227 & 255 & 134 & 111 & 140 & 40 & 31 & 23 & 1.393 \\
\hline Hymenoptera & 11 & 8 & 9 & 13 & 14 & 16 & 19 & 3 & 5 & 3 & 101 \\
\hline Lepidoptera & 5 & 7 & 5 & 4 & 20 & 19 & 12 & 1 & 5 & 2 & 80 \\
\hline Collembola* & - & - & - & - & 13 & 11 & 23 & 12 & 7 & 9 & 75 \\
\hline Coleoptera & 2 & 4 & 3 & 3 & 1 & 3 & 7 & - & 2 & 4 & 29 \\
\hline Homoptera & 3 & 1 & 1 & 2 & - & 4 & 2 & 2 & - & - & 15 \\
\hline Thysanoptera & 3 & 3 & 2 & 1 & - & - & 2 & 1 & - & - & 12 \\
\hline Trichoptera & 3 & 1 & 2 & 2 & - & - & 1 & - & - & - & 9 \\
\hline Hemiptera & - & 1 & - & 2 & - & - & 1 & 1 & 1 & - & 6 \\
\hline Dermaptera & - & 1 & - & - & - & 2 & - & - & 1 & 1 & 5 \\
\hline Orthoptera & - & - & 1 & - & - & 1 & - & - & - & 2 & 4 \\
\hline Total & 238 & 247 & 250 & 282 & 182 & 167 & 207 & 60 & 52 & 44 & 1.729 \\
\hline
\end{tabular}

*Subclasse da classe Insecta. 
Tabela 2 - Freqüência das ordens de Insecta capturadas no período de incubação de Lentinula edodes (shiitake), cultivado em toros de eucalipto, no Município de Arroio do Padre, RS, 2005.

\begin{tabular}{lccc}
\hline \multicolumn{4}{c}{ Freqüência da ordem Insecta } \\
\hline Ordens & $\begin{array}{c}\text { Absoluta } \\
\left(\mathrm{n}^{\circ}\right)\end{array}$ & $\begin{array}{c}\text { Relativa } \\
(\%)\end{array}$ & $\begin{array}{c}\text { Acumulada } \\
(\%)\end{array}$ \\
\hline Diptera & 1.393 & 80,57 & 80,57 \\
Hymenoptera & 101 & 5,84 & 86,41 \\
Lepidoptera & 80 & 4,63 & 91,04 \\
Collembola* & 75 & 4,34 & 95,38 \\
Coleoptera & 29 & 1,68 & 97,06 \\
Homoptera & 15 & 0,87 & 97,93 \\
Thysanoptera & 12 & 0,69 & 98,62 \\
Trichoptera & 9 & 0,52 & 99,14 \\
Hemiptera & 6 & 0,35 & 99,49 \\
Dermaptera & 5 & 0,29 & 99,78 \\
Orthoptera & 4 & 0,23 & 100,00 \\
\hline Total & 1.729 & 100,00 \\
\hline
\end{tabular}

*Subclasse da classe Insecta.

As ordens Diptera, Hymenoptera, Lepidoptera e Coleoptera, bem como a subclasse Collembola, nesta seqüência, apresentaram as maiores freqüências de espécimes coletados (Tabela 2).

NaordemDiptera destacou-seasmoscas pertencentes à família Sciaridae, com um número de espécimes mais significativamente detectado, comprovando citações de Figueiredo; Mucci (1995) e de Al-Amidi (1995). Segundo MenZel;Mohrig (1997) esta família apresenta uma dispersão por quase todo o mundo. Suas larvas são conhecidas por se alimentar de matéria orgânica em decomposição, plantas e "spawn". Espécimes dessa família são conhecidos também por causar grandes prejuízos na produção mundial decogumelos. Uma das maiores pragas na produção de cogumelos na América do Norte tem sido causada por espécies de Sciaridae, devido ao hábito das fêmeas que depositam seus ovos sobre o substrato para dar continuidade ao seu ciclo de vida. Os adultos desta família não são atraídos pelo cogumelo e sim, pela qualidade do "spawn", pois um substrato bem colonizado inibe a ovoposição defêmeas de Sciaridae (Russey; GuRneY, 1968).

Os espécimes desta família ocorreram em alta freqüência, principalmente nas 4 primeiras coletas (Tabela 3).

A maior diferença do número de insetos capturados nas primeiras quatro coletas, em relação à diminuição nas demais, pode estar relacionada ao ciclo de vida destes insetos, bem como as influências climáticas do local da realização do trabalho. Por outrolado, deve-se considerar também algum efeito decorrente da pulverização feita com o extrato de cinamomo.
Tabela 3 - Freqüência absoluta e relativa de exemplares da família Sciaridae, ordem Diptera, classe Insecta, capturados no período de incubação de Lentinula edodes (shiitake), cultivado em toros de eucalipto, no Município de Arroio do Padre, RS, 2005.

\begin{tabular}{lcc}
\hline Coletas & \multicolumn{2}{c}{ Freqüência da família Sciaridae } \\
\cline { 2 - 3 } & Absoluta $\left(\mathrm{n}^{\circ}\right.$.) & Relativa $(\%)$ \\
\hline 1 & 190 & 17,51 \\
2 & 201 & 18,53 \\
3 & 207 & 19,08 \\
4 & 234 & 21,57 \\
5 & 71 & 6,54 \\
6 & 41 & 3,78 \\
7 & 84 & 7,74 \\
8 & 18 & 1,66 \\
9 & 20 & 1,84 \\
10 & 19 & 1,75 \\
\hline Total & 1.085 & 100,00 \\
\hline
\end{tabular}

A freqüência de alguns insetos no cultivo de cogumelos já foi constatada no Brasil, conforme observado numa produção de shiitake em toros de eucalipto, no Município deSão Carlos, SP,ZORZENON;POTENZA (2003) constataram que em média $35,17 \%$ dos toros inoculados e em fase de produção, das 15 propriedades amostradas, encontravam-se infestados pela traça Opogona sacchari (Lepidoptera: Tineidae), a qual, segundo os mesmos autores, tem sido constatada, inviabilizando cultivos de cogumelos conduzidos por pequenos e médios produtores, pois se trata de um inseto de ampla distribuição e com inúmeros hospedeiros.

Ao coletar o cogumelo Amylocystis lapponica, em ambiente de floresta, KOMONEN (2001) verificou que este apresentava uma comunidade de 60 espécies de insetos, sendo 37 de Coleoptera, 13 de Diptera, 8 de Hymenoptera e 2 de Homoptera. Estas duas últimas ordens foram diferentes das encontradas neste trabalho, sendo as primeiramente citadas também encontradas durante o cultivo de shiitake em toros de eucalipto. Isto sugere que estes insetos se desenvolvem em cogumelos nativos e podem passar para os que são cultivados.

\section{CONCLUSÕES}

De acordo com os dados obtidos comprovou-se que durante a fase de incubação da cultura de shiitake (Lentinula edodes), cultivado em toros de Eucalyptus spp., ocorre uma grande diversidade de espécies de insetos, distribuídas em nove ordens, entre as quais predominam a classe Diptera, com a maior freqüência 
das espécies pertencentes à família Sciaridae, na qual estão registradas as pragas mais severas do cultivo de cogumelos.

\section{REFERÊNCIAS}

Al-Amidi, A.H.K. Occurrence of insects and mites in mushroom compost in Ireland. In: ElLIOTT, T.J. (Ed.). Science and cultivation of edible fungi. Roterdam: Balkmam, 1995. p.539-546.

Bononi, V.L.R.; Capelari, M; Maziero R.; Trufem, S.F.B. Cultivo de cogumelos comestíveis. São Paulo: Icone, 1995. p.95-104.

Bonham, K.J.; Mesibov, R.; BASHFORD, R. Diversity and abundance of some ground-dwelling invertebrates in plantation vs. native forests in Tasmania, Australia. Forests Ecology and Management, v.158, n.1/3, p.237247, 2002.

Bueno, F.S.; Eira, A.F.; Furlan, M.; Neto, P.F. Natural and organo-synthetic products to controlLentinulaedades competitors on Eucalyptus saligna logs. In: RoMAINERINKER, C.P. (Ed.). Science and Cultivation of Edible and Medicinal Fungi. Miami: Pensylvania University Press, 2004. p.431-439.

Chang,S.T.;Miles P.G. Edible mushrooms and their cultivation . Boca Raton: CRC Press, 1989. 345p.

CHANG, S.T. Production of cultivated edible mushroom in China with emphasis on Lentinula edodes. ISMS Newsletter, v.4, p.3-6, 1999.

CHANG, S.T. Past and present trends in the production of Lentinula edodes in Asia. In: INTERNATIONAL CONFERENCE OF MUSHROOM, 4., 2002, Cuernavaca. Proceedings. Cuernavaca, 2002, p.1-8.

Donoghue, J.D.; Denison, W.C. Shiitake cultivation: gas phase during incubation influences productivity. Mycologia, v.87, n.2, p.239-244, 1995.

EIRA, A.F.; MNHONI, M.T.A. Manual teórico-prático do cultivo de cogumelos comestíveis .2.ed. Botucatu:FundaçãodeEstudos e Pesquisas Agrícolas e Florestais/UNESP, 1997. 115p.

Figueiredo, M.B.; MuccI, E.S.F. Doenças e pragas do cogumelo comestível (Agaricus campestris L. EX FR.), In: ENCONTRO NACIONAL SOBRE COGUMELOS COMESTÍVEIS, 1., 1980, Mogi das Cruzes. Anais. São Paulo: Instituto de Botânica, 1985. p.69-91.

Gaston, K.J.; BLACKBURN, T.M.; GREENWOOD, J.J.D.; GREGORY, R.D.; QuinN, R.M.; Lawston, J.H. Abudance - occupancy relationships. Journal of Applied Ecology, v.37, p.39-59,2000.

Jactel, H.; Goulart, M.; Menassieu, P.; Goujon, G. Habitat diversity in forest plantations reduces infestations of the pine stem boner Diocyctria sylvestrella. Journal of Applied Ecology, v.39, p.618-628, 2002.

Komonen, A. Structure of insect communities inhabiting old-growth forest specialist bracket fungi. Ecological Entomology, v.26, p.63-75, 2001.
LARANJEIRO, A.J. Estabilidade da entomofauna num mosaico de plantação de eucalipto e áreas naturais de conservação. 2003. 142p. Tese (Doutorado em Ciências) - Escola Superior de Agricultura "Luiz de Queiroz", Universidade de São Paulo, Piracicaba, 2003.

Leatham, G.F. Growth and development of Lentinusedodes on a chemically defined medium. In: MOORE, D.; Casselton, L.A.; Wood. D.A.; Frankland, J.S. (Eds.). Developmental biology of higher fungi. Cambridge: Cambridge Press, 1985. p.4003-4027.

Mata, G.; Savoie, J.M.; Delpech, P.; Olivier, J.M. Reductions in the incidence of Trichoderma spp. using substrat supplementation with peat and an alternative spawn during cultivation of Lentinula edodes on pasteurized wheat straw. Agriculture and Environment, v.18, p.51520, 1998 .

Menzel F.; Mohrig W. Family Sciaridae. In:Papp, L.; Darvas, B. (Eds.).Manual of palaearctic Diptera. Budapest:Science Herald, 1997. v.2, n.69, p.51.

Ore, P. Manual on mushroom cultivation. Amsterdam: Tool Foundation, 1991. 247p.

Pinto, R.; Junior, J.S.Z.; Ferreira, J.A.M.; Zanuncio, J.C. Flutuação populacional de coleóptera em plantio de Eucalyptus urophylla no município de Três Marias, Minas Gerais. Floresta e Ambiente, v.7, n.1, p.143-151, 2000.

Przybylowicz, P.; Donoghue, J. Shiitake growers handbook: The art and science of mushroom cultivation. Dubuque: Kendall, 1990. 217p.

Quimio, T.H.; ChANG, S.T.; RoYse, D.J. Technical guidelines for mushroom growing in the tropics. Rome: FAO, 1990. $155 \mathrm{p}$.

Royse, D.J.; Schisler, L.C.; Dehle, D.A. Shiitake mushrooms: consumption, production and cultivation. Interdisciplinary Science Reviews, v.10, p.329-335, 1985.

RusSEY, N.W.; GuRNEY, B. Biology and control of theSciaridae Lycoriella auripila Winn. (Diptera: Lycoriidae) in mushroom culture. Annals of Applied Biology, v.62, p.395-403, 1968.

SAвотA, C. Strain of shiitake mushroom [Lentinula edodes (Berk.) Pegler] and wood species affect the yield of shiitake mushrooms. Hort Technology, v.6, p.388-393, 1996.

ZoRZENON, F.J.; Potenza, M.R. Diagnóstico de danos causados pela traça Opogona sacchari (Lepidoptera:Tineidae) em cultivo de shiitake (Lentinula edodes). Arquivos do Instituto Biológico, São Paulo, v.70, n.4, p.507-509, 2003. Disponível em: <http:/ /www.biologico.sp.gov.br/ ARQUIVOS/V70_4/zorzenon.pdf>. Acesso em: 15 jan.2005.

Recebido em 10/7/06

Aceito em 30/10/06 